\title{
小分子生物硫醇荧光探针研究进展
}

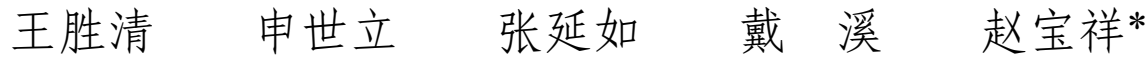 \\ (山东大学化学与化工学院 济南 250100)
}

\begin{abstract}
摘要 半胱氨酸 $(\mathrm{Cys}) 、$ 同型半胱氨酸 $(\mathrm{Hcy})$ 和还原型谷胱甘肽 $(\mathrm{GSH})$ 等小分子生物硫醇在人的生理活动中起着重要作用. 近年来, 生物和环境样品中小分子生物硫醇的检测引起科学家们极大的兴趣, 生物硫醇苂光探针和比色传感器得到快 速发展. 根据探针与生物硫醇的反应机理，包括利用小分子生物硫醇中颈基与探针反应、巯基和氨基协同与探针反应, 综述两年来生物硫醇小分子荧光探针的设计、合成与应用进展.

关键词 苂光; 探针; 还原型谷胱甘肽; 同型半胱氨酸; 半胱氨酸; 生物硫醇
\end{abstract}

\section{Recent Progress in Fluorescent Probes for the Detection of Biothiols}

\author{
Wang, Shengqing Shen, Shili Zhang, Yanru Dai, Xi Zhao, Baoxiang* \\ (Institute of Organic Chemistry, School of Chemistry and Chemical Engineering, Shandong University, Jinan 250100)
}

\begin{abstract}
Intracellular thiols, such as cysteine (Cys), homocysteine (Hcy) and glutathione (GSH), play crucial roles in maintaining biological systems. In last decade, developing small molecule probes for selective and sensitive detection of thiols under physiological conditions have attracted great interest. Fluorescent and colorimetric sensors are classified into two types of reactions according to their mechanism of reaction with thiols, including the reaction with mercapto group, the reaction with mercapto and amino group. Therefore, in this review we provide a general overview of the selected recent research involving the design and application of biological thiol-selective chemosensors.
\end{abstract}

Keywords fluorescence; probe; glutathione; homocysteine; cysteine; thiol

半胱氨酸(Cys)、同型半胱氨酸(Hcy)和还原型谷胱 甘肽(GSH)等生物硫醇在人的生理活动中起着重要作 用 ${ }^{[1 ~ 8]}$. 半胱氨酸是谷胱甘肽、乙酰辅酶和牛磺酸的前 体，同时它也是生物体硫铁络合物中硫配体的提供者. 人体中的半胱氨酸水平异常会引起生长缓慢、毛发色素 脱色、水肿、嗜睡、肝功能损伤、肌肉松弛和肥胖、皮 肤松弛、身体虚弱等症状 ${ }^{[0 ~ 11]}$. 人体中同型半朕氨酸浓 度过高可能引起心血管病和阿尔茨海默氏病, 同型半胱 氨酸在血浆中的总浓度与某些先天性疾病和老年认知 障碍有关 ${ }^{[12 ~ 14]}$. 细胞内最多的非蛋白統基谷胱甘肽, 在 维持细胞生理功能中起着重要作用, 包括细胞内的氧化 还原反应、异物代谢、信号传导与基因调控等. 谷胱甘 肽是细胞内最多的小分子生物硫醇 $(1 \sim 10 \mathrm{mmol} / \mathrm{L})$, 还 原型谷胱甘肽与氧化型谷胱甘肽之间存在着氧化还原 平衡. 还原型谷胱甘肽在细胞的生长、维持细胞正常功 能的氧化还原平衡上起着关键作用. 此外, 人体中还原 型谷胱甘肽浓度异常与癌症、阿尔茨海默氏症和心血管 等疾病有关 ${ }^{[15 ~ 19]}$. 因此, 生物和环境样品中小分子生物
硫醇的检测引起广泛关注.

经典的检测小分子生物硫醇的方法, 如电化学检测 法与高效液相色谱法, 只能检测生物硫醇的总量, 并且 需要大量的样品才能检测准确. 因此, 方便廉价、能够 定性定量分析生物硫醇, 并且可以对生物样品进行实时 检测的方法非常重要. 如同荧光分析法在检测金属离 子[20 31] 与溶液 $\mathrm{pH}^{[32 \sim 39]}$ 获得广泛应用一样, 在众多检测 生物硫醇的方法中，荧光分析法操作简单、检测限低和 易于操作, 受到广泛关注. 自从 Sippel ${ }^{[40]}$ 报道了利用硫 醇与马来酰亚胺片段发生加成反应的原理设计的化合 物, $N$-(4-(7-二乙氨基-4-甲基香豆素-3-基)苯基)马来酰 亚胺，作为最早的检测小分子硫醇的探针之一，小分子 生物硫醇探针的设计与合成取得了巨大成就. 生物硫醇 苂光探针在生物体中的应用主要分为 3 个阶段：一是在 细胞内苂光成像; 二是活体组织成像; 三是动物活体体 内成像 ${ }^{[41 ~ 44]}$.

根据探针与生物硫醇的反应机理, 可以把探针分为 两大类. 第一类主要是利用生物硫醇分子中颈基的亲核

*E-mail: bxzhao@sdu.edu.cn

Received March 12, 2014; revised April 25, 2014; published online May 7, 2014. 
性、还原性和金属离子络合能力与探针分子发生反应， 反应类型包括: 迈克尔加成反应、亲核取代反应、磺酰 胺与磺酰酯断裂反应、颈基与二硫键交换反应、颈基与 酯反应、颈基与硒氮键反应、颈基与呋喃环加成反应、 颈基与碳氮双键加成反应、統基还原偶氮化合物反应、 颈基与金属配合物反应. 该类型的生物硫醇荧光探针设 计容易, 但是很难把半胱氨酸、同型半胱氨酸和还原型 谷胱甘肽进行区分. 第二类荧光探针设计主要是利用荧 光探针与生物硫醇中颈基和氨基官能团协同反应, 主要 包括半胱氨酸或同型半胱氨酸的共轭加成环化反应、半 胱氨酸或同型半胱氨酸与醛成环反应、半胱氨酸与醛基 缩合再与双键加成成环反应、半胼氨酸发生分子内亲核 取代反应、亲核取代-加成成环反应. 该类生物硫醇荧光 探针可以把半胱氨酸、同型半胱氨酸与还原型谷胱甘肽 进行区分, 或者是单独识别三者中某一个. 第二类探针 与第一类探针相比, 设计难度大, 文献相对较少. 本文 从以上两点出发综述两年来生物硫醇小分子探针的设 计、合成与应用进展.

\section{1 小分子生物硫醇中俆基与探针反应}

颈基是半胱氨酸区分其它氨基酸的典型官能团, 利 用颈基的亲核性设计探针, 检测小分子生物硫醇, 是经 典方法，也是目前广泛采用的设计基础.

\section{1 基于生物硫醇分子与探针发生加成反应的探针}

$\alpha, \beta$-不饱和羰基化合物与生物硫醇的颈基发生亲核 加成反应，广泛用于探针设计. 1970 年, Kanaoka 等 ${ }^{\left[{ }^{[5]}\right.}$ 利 用马来酰亚胺与颈基发生亲核加成反应设计检测小分 子生物硫醇的化学剂量计.

Youziel 等 ${ }^{[46]}$ 报道了基于丹黄酰氯荧光基团的荧光 探针 1,2 和 3 , 用于检测小分子生物硫醇. 在马来酰亚 胺片段中的 3 或 4 号位置引入卤素或者硫醚化合物, 使 丹黄酰氯荧光基团产生淬灭, 在生物硫醇小分子存在 下, 硫醇中的巯基与马来酰亚胺片段发生加成反应, 使 丹黄酰胺与马来酰亚胺的硫醚键断裂, 生成小分子生物 硫醇与马来酰亚胺的硫醚化合物和丹磺酰胺的二硫键 化合物 $\mathbf{3}^{\prime}$, 从而使荧光增强. 探针 3 可用于检测 HEK 细 胞内的生物硫醇.

Liu 等 ${ }^{\left[{ }^{47}\right]}$ 报道了基于苯并噁嗪酮-半花菁生物硫醇 荧光比率探针 4. 半花菁结构导致易与硫醇的颈基发生 迈克尔加成反应, 生成硫醚化合物. 在 $\mathrm{CH}_{3} \mathrm{CN}-\mathrm{Tris}-\mathrm{HCl}$ 缓冲溶液中 $(50 \mathrm{mmol} / \mathrm{L}, \mathrm{pH} 7.4,1: 4, V / V)$, 探针 4 与半 胱氨酸作用, 在紫外-可见与荧光光谱中分别发生蓝移 143 和 $109 \mathrm{~nm}$, 颜色由黄色变为蓝色. 导致长波 $679 \mathrm{~nm}$ 发射荧光强度逐渐降低, 短波 $570 \mathrm{~nm}$ 发射荧光强度逐 渐增强, 两者成比例关系, 适宜 $\mathrm{pH}$ 范围在 3.0 与 8.5 之
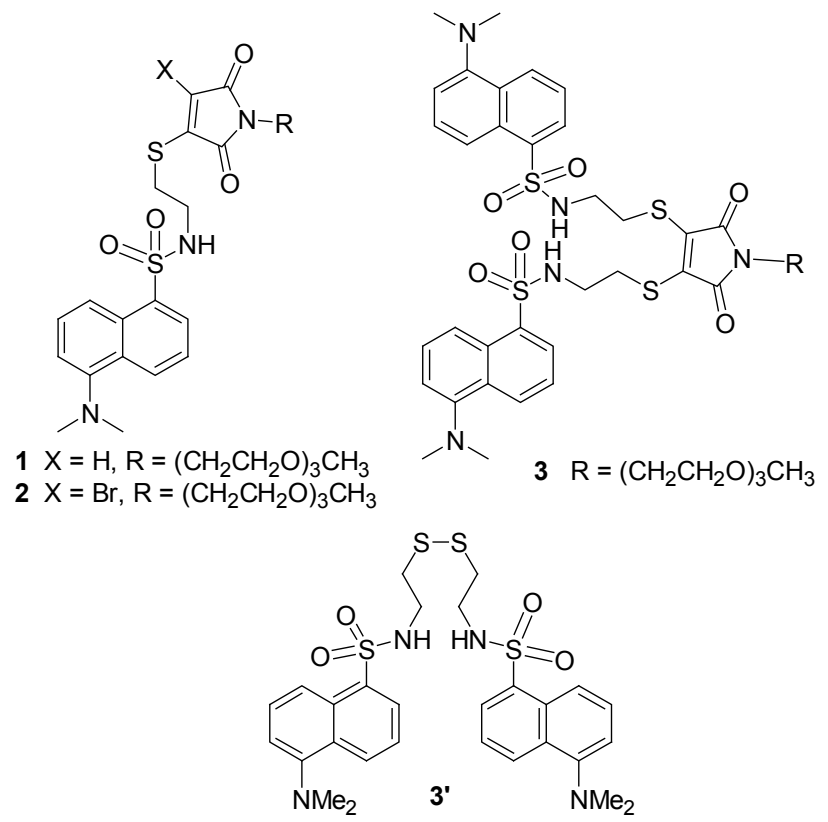

间，探针 4 可以用于人口腔表皮样癌细胞(KB)活体细胞 中荧光成像检测生物硫醇.

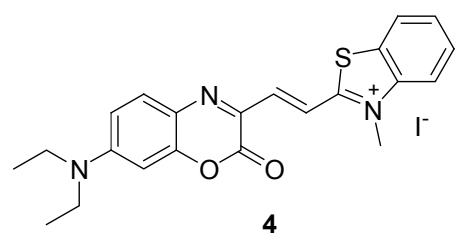

Zhou 等 ${ }^{[8]}$ 报道了以香豆素为荧光基团的离子型探 针 5 , 由于季铵盐的强吸电子效应, 有利于发生迈克尔 加成反应. 电子效应与空间效应的协同作用使得探针 $\mathbf{5}$ 对半胱氨酸具有独特的选择性, 可以有效地区分半胱氨 酸与还原型谷胱甘肽、同型半胱氨酸. 该探针可以在纯 磷酸盐缓冲溶液( $\mathrm{PBS})(\mathrm{pH}=7.4)$ 对半胱氨酸做出响应, 抗干扰能力强, 最低检测限为 $25 \mathrm{nmol} / \mathrm{L}$. 此外, 探针 $\mathbf{5}$ 可以用来检测小牛血清中的 $\mathrm{Cys}_{34}$, 以及 $\mathrm{HeLa}$ 细胞中的 小分子生物硫醇. 尤其难得的是, 对于具有 3 个二硫键 而不含半胱氨酸的胰岛素，探针不与之发生反应，但是 加入二硫苏糖醇破坏胰岛素中的二硫键后探针 $\mathbf{5}$ 的荧光 信号增强.<smiles>CCN(CC)c1ccc2cc(C(=O)/C=C/c3ccc[n+](C)c3)c(=O)oc2c1</smiles>

Yang 等 ${ }^{[49}$ 报道了以香豆素为发光基团的荧光探针 6, 用来检测含有颈基的生物小分子. 在 4-羟乙基哌嗪 乙磺酸(HEPES)缓冲溶液中 $(10 \mathrm{mmol} / \mathrm{L}, \mathrm{pH} 7.4)$, 化合 物 6 中的 $\alpha, \beta$-不饱和酮的烯双键与含有颈基的小分子化 合物发生迈克尔加成反应, 生成硫醚, 苂光增强, 紫外- 
可见吸收峰发生红移 $52 \mathrm{~nm}$. 探针 6 对活性硫醇分子具 有很好的灵敏性, 对半胱氨酸、同型半胱氨酸和还原型 谷胱甘肽的最低检测线分别为 $0.18,0.82$ 和 $0.70 \mathrm{~mol} / \mathrm{L}$, 在 $\mathrm{pH}=7.4$ 时, 探针与半胱氨酸, 同型半胱氨酸和谷胱 甘肽作用的速率常数分别为 1272,850 和 $423 \mathrm{~L} \cdot \mathrm{mol}^{-1}$ • $\mathrm{s}^{-1}$. 探针 6 可以检测 $\mathrm{HePG} 2$ 细胞中的半胱氨酸, 并且成 功地检测人体血浆中的小分子生物硫醇.

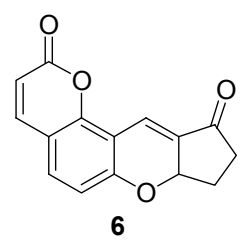

$\mathrm{Wu}$ 等 ${ }^{[50]}$ 合成的双光子比例荧光探针 7 , 在乙醇-磷 酸盐缓冲溶液中 $(V / V=1: 4, \mathrm{pH} 7.4)$, 加入半胱氨酸、同 型半胱氨酸与还原型谷胱甘肽后, 荧光强度 $I_{485} / I_{590}$ 的 比值分别增强 23, 12 和 28 倍. 探针 7 与半胱氨酸、同型 半胱氨酸与还原型谷胱甘肽作用的二级反应速率常数 分别为 $14.9,4.3$ 和 $7.8 \mathrm{~m}^{-1} \cdot \mathrm{s}^{-1}$. 与半胱氨酸作用时, 荧 光发射波长由 $572 \sim 725 \mathrm{~nm}$ 蓝移到 $425 \sim 572 \mathrm{~nm}$, 蓝移 波长超过 $100 \mathrm{~nm}$, 最低检测限为 $6 \mathrm{~mol} / \mathrm{L}$. 探针 7 用于 MDA-MB-231 细胞与牛血清中生物硫醇的细胞内成像.

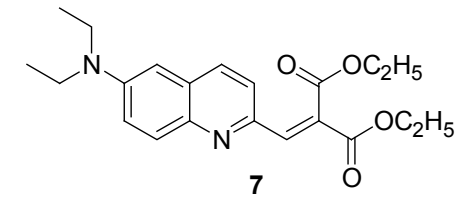

Wang 课题组 ${ }^{[1]}$ 合成了以荧光素为苂光基团、硝基 烯为识别基团的小分子生物硫醇探针 8 . 当加入半胱氨 酸后, 紫外-可见光吸收波谱发生蓝移, 最大吸收波长 为 $497 \mathrm{~nm}$, 苂光发射波长在 $520 \mathrm{~nm}$ (Eq. 1). 半胱氨酸 与探针的硝基烯发生迈克尔加成反应导致苂光增强与 紫外-可见吸收蓝移. 探针对半胱氨酸作用响应迅速、时 间短, 速率常数为 $2.3 \mathrm{~min}^{-1}$, 最低检测线为 $0.2 \mathrm{~mol} / \mathrm{L}$. 探针 8 用于活细胞 PC-12 中生物硫醇的检测.<smiles></smiles>

Long 等 ${ }^{[52]}$ 报道的以香豆素为荧光母体的比例苂光
探针 9 可以在含有 $2 \%$ 二甲基亚砜(DMSO)的磷酸盐缓冲 溶液中 $(10 \mathrm{mmol} / \mathrm{L}, \mathrm{pH}=7.4)$ 检测小分子生物硫醇( Eq. 2). 在测试条件下探针 9 没有荧光(荧光量子产率 $<0.0001$ ), 加入半胱氨酸后, 荧光增强 246 倍, 对半胱 氨酸的最低检测限为 $0.22 \mathrm{~mol} / \mathrm{L}(S / N=3)$. 探针 9 具有 很好的选择性, 可以区分小分子生物硫醇与其它的生物 试剂. 探针 9 与与半胱氨酸、同型半胱氨酸和还原型谷 脱甘肽的反应速率常数分别为 $1.86,0.67$ 和 $0.52 \mathrm{~min}^{-1}$, 可用于胰腺癌细胞与人体血清的生物硫醇成像.<smiles>[R]CC(c1c(O)ccc2cc(C(=O)OCC)c(=O)oc12)C(C(C)=O)C(=O)CC</smiles>

\section{2 基于放基亲核取代反应的荧光探针.}

尽管有许多检测生物硫醇的研究, 但是, 由于结构 与化学反应性质非常相似, 区分谷胱甘肽, 半胱氨酸和 同型半胱氨酸面临巨大挑战. 开发具有高选择性的苂光 探针仍然是巨大的挑战.

Zhang 等 ${ }^{[53]}$ 报道了在甲醇/HEPES $(V: V=7: 3$, $\mathrm{pH}=7$ ) 缓冲溶液中识别 $\mathrm{Cys} / \mathrm{Hcy}$ 的探针 10. 加入半胱氨 酸或者同型半胱氨酸后, 探针 $\mathbf{1 0}$ 在紫外-可见光谱中 $430 \mathrm{~nm}$ 处的吸光度逐渐降低, $580 \mathrm{~nm}$ 处的吸光度逐渐增 加, 等吸收点出现在 $465 \mathrm{~nm}$ 处, 溶液的颜色由黄绿色转 变为红棕色. 荧光光谱中, 探针 $\mathbf{1 0}$ 与半胱氨酸或者是同 型半胱氨酸作用后在 $588 \mathrm{~nm}$ 处荧光强度迅速增加(Eq. 3). 另一方面，还原型谷胱甘肽由于空间位阻原因与探 针反应活性降低, 在 $1 \mathrm{~h}$ 之内荧光变化小. 该探针可用 于活细胞的细胞成像.

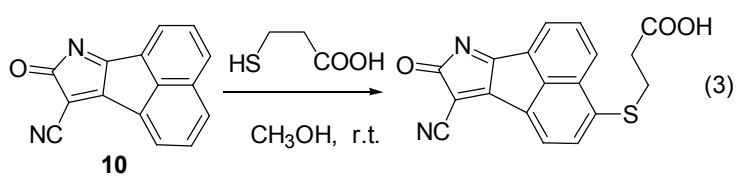

$\mathrm{Niu}$ 等 $^{[54]}$ 设计合成了基于硼 -二吡咯亚甲基 (BODIPY)为荧光基团的荧光比例探针 11. 利用颈基的 亲核性，通过亲核取代反应生成硫醚，有效地、选择性 地把谷胱甘肽与半胼氨酸和同型半胱氨酸进行区分. 半 胱氨酸与同型半胱氨酸中統基先与探针 $\mathbf{1 1}$ 发生亲核取 代反应，然后再发生分子内氨基亲核取代反应; 还原型 谷胱甘肽只能颈基发生亲核取代反应，由于空间原因氨 基不会发生分子内亲核取代反应(Eq. 11). 探针 11 中的 氯原子被硫原子或氮原子取代后产物的最大发射波长 
不同, 所以可以把还原型谷胱甘肽与半胱氨酸和同型半 胱氨酸进行区分. 在含 $5 \%$ 乙腈的 HEPES 缓冲水溶液中 $(20 \mathrm{mmol} / \mathrm{L}, \mathrm{pH}=7.4)$, 苂光强度与 $0 \sim 60 \mathrm{~mol} / \mathrm{L}$ 范围内 的谷胱甘肽浓度成比例, $R=0.993$, 最低检测线为 $8.6 \times$ $10^{-8} \mathrm{~mol} / \mathrm{L}$. 探针 11 对 HepG2 细胞中谷胱甘肽比例成 像.

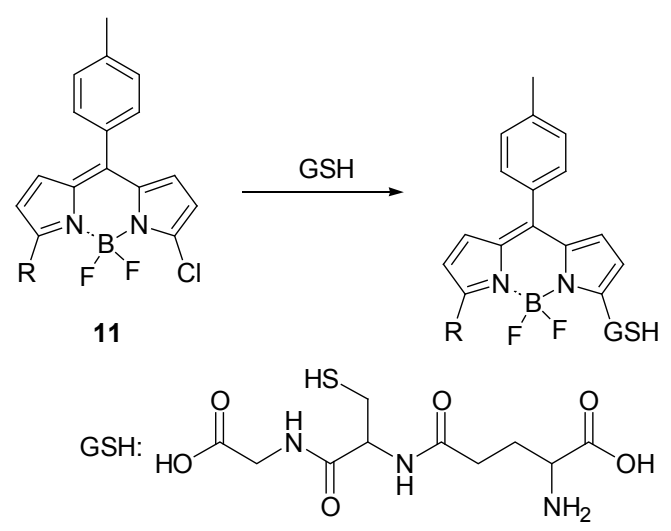

Zhang 等 ${ }^{[55]}$ 报道了以 1,8 -荟碳酰亚胺为发色团的比 色比例探针 12. 探针 12 荎环上的溴原子通过芳香环的 亲核反应被半胱氨酸的巯基取代, 紫外-可见光谱红移 $58 \mathrm{~nm}$ (Eq. 5). 在乙醇与 HEPES 缓冲溶液 $(V: V=7: 3$, $\mathrm{pH}=7.3)$ 中加入半胱氨酸后颜色由无色变为黄色, 在 $\mathrm{pH}=3 \sim 11$ 范围内对半胱氨酸有选择性响应, 对半胱氨 酸的最小检测浓度为 $50 \mathrm{~mol} / \mathrm{L}$.
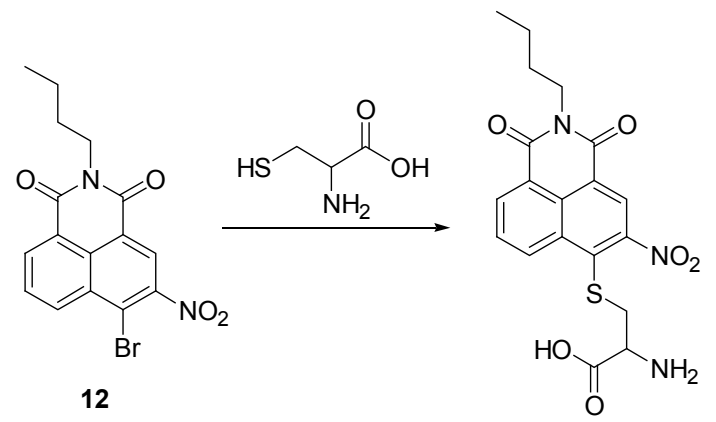

\section{3 基于硫醇使磺酰胺或者磺酸酯断键的荧光探针}

Tang 等 ${ }^{[56]}$ 报道的磷光探针 $\mathbf{1 3}$, 发光基团是三价金 属铱与配体形成的金属络合物. 探针 13 在 $\mathrm{CH}_{3} \mathrm{CN} / \mathrm{H}_{2} \mathrm{O}$ $(V: V=4: 1, \mathrm{pH} 7.2)$ 的混合溶液中 $\left(4.07 \times 10^{-5} \mathrm{~mol} / \mathrm{L}\right)$ 与半胱氨酸作用后, 紫外-可见吸收光谱中 $250 \sim 550$ $\mathrm{nm}$ 处的吸收峰逐渐增强, 说明探针 13 与半胱氨酸发生 了反应(Eq. 6); 在苂光谱图中, 探针 13 没有发射峰, 当 加入半胱氨酸以后, 在 $603 \mathrm{~nm}$ 处出现了 1 个发射峰, 这 个发射峰与脱除 2,4-二硝基磺酰基以后形成酚的图谱一 致，同样的，质谱与核磁氢谱都验证了脱除 2,4-二硝基 磺酰基的机制. 半胱氨酸的类似物同型半胱氨酸具有相 同的现象, 而位阻增大的还原型谷胱甘肽在相同条件下
与探针 13 几乎不发生作用. 探针 13 可用于 HeLa 细胞 检测生物硫醇.

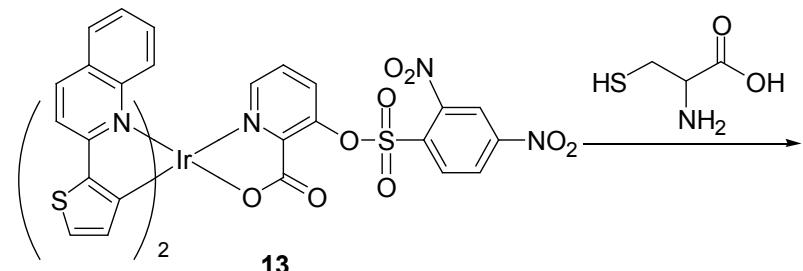

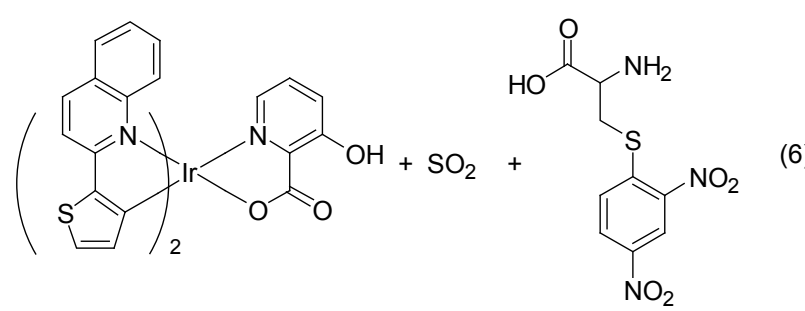

Maity 等 ${ }^{[57]}$ 报道了增强型近红外菼光探针 14. 在 $\mathrm{pH}$ 为 7.4, 浓度为 $10 \mathrm{mmol} / \mathrm{L}$ 的磷酸盐缓冲溶液中, 探 针 14 与还原型谷胱甘肽作用使 2,4-二硝基苯磺酰基被 硫醇断裂后, 紫外可见吸收谱图中 $390 \mathrm{~nm}$ 处的吸收峰 迅速消失, 476 和 $581 \mathrm{~nm}$ 出现两个新的吸收峰(Eq. 7); 苂光谱图中在近红外区域 $695 \mathrm{~nm}$ 处出现发射峰，斯托 克斯位移为 $119 \mathrm{~nm}$. 溶液颜色由亮绿色变为蓝色. 在 pH 6 10 范围，探针 14 对谷胱甘肽选择性响应，可以 有效地检测小牛血清中的小分子生物硫醇 ${ }^{[58,59]}$.

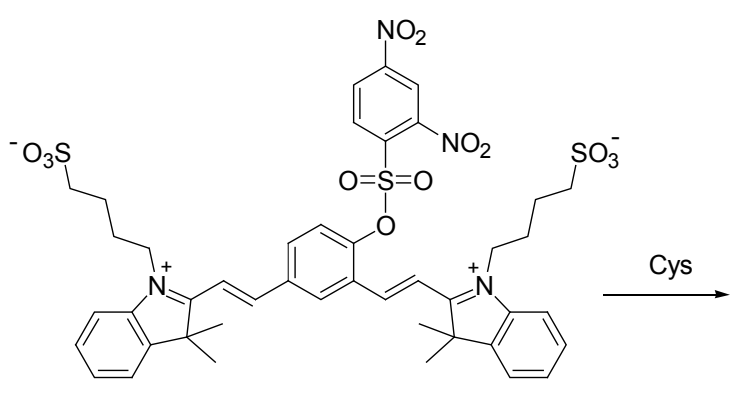

14

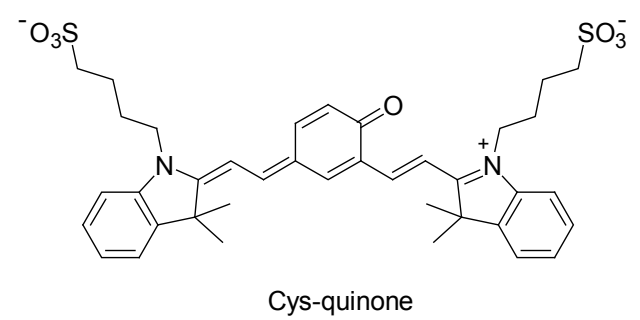

(7)

Wei 等 ${ }^{[60}$ 报道了以 7 -氨基香豆素为苂光团的苂光 探针 15, 检测还原型谷胱甘肽与半胱氨酸. 苂光探针具 有微弱的苂光, 在 $45{ }^{\circ} \mathrm{C}, \mathrm{pH}=9.0$ 的磷酸盐的缓冲溶液 中加入还原型谷胱甘肽或者半胱氨酸后荧光增强. 不论 是半胱氨酸还是谷胱甘肽在浓度为 $30 \sim 60 \mathrm{~mol} / \mathrm{L}$ 范围 
内苂光强度与被测物浓度成线性关系. 探针 15 可以在 体内和体外用来检测 GSH 和半胱氨酸(Eq. 8). 该探针抗 干扰能力强, 对 GSH 和半胱氨酸具有良好的选择性, 可 以把 $\mathrm{GSH}$ 和半胱氨酸与其它氨基酸、金属离子和蛋白 质进行区分. 但是, 高于室温甚至高于体温的应用条件 $\left(45{ }^{\circ} \mathrm{C}\right)$ 尚待改善. 探针可以检测活体 Hela 细胞中的 $\mathrm{GSH} /$ 半胱氨酸.<smiles>[R5][Sb](C)(C)C</smiles><smiles>[R5]c1ccc([N+](=O)[O-])cc1[N+](=O)[O-]</smiles>

Wang 等 ${ }^{[61]}$ 报道了以 $1,3,5$-三芳基吡唑啉为苂光团 的荧光探针 16, 检测还原型谷胱甘肽(Eq. 9). 探针 16 是 以光电子诱导转移机理设计合成的增强型探针. 探针 16 在室温、乙腈-PBS $(10 \mathrm{mmol} / \mathrm{L}, \mathrm{pH}=7.4, V: V=3$ : 7)的缓冲溶液中加入还原型谷胱甘肽或者半胱氨酸后 最大发射峰在 $464 \mathrm{~nm}$ 荧光强度增强. 由于在生物体中 还原型谷胱甘肽的浓度为 $(1 \sim 10 \mathrm{mmol} / \mathrm{L})$, 是半胱氨酸 浓度的千倍以上, 所以探针 16 在生物中主要是选择还 原型谷胱甘肽. 谷胱甘肽在浓度为 $10 \sim 180 \mathrm{~mol} / \mathrm{L}$ 范围 内苂光强度与被测物浓度成线性关系, 对还原型谷胱甘 肽的最小检测浓度为 $4.11 \times 10^{-7} \mathrm{~mol} / \mathrm{L}$. 探针 16 对还原 型谷胱甘肽作用的 $\mathrm{pH}$ 值范围广为 $4 \sim 10$, 但是作用时 间长为 $8 \mathrm{~h}$. 共聚焦显微镜成像证实探针 16 可用于检测 A549 细胞和小牛血清中的小分子生物硫醇.

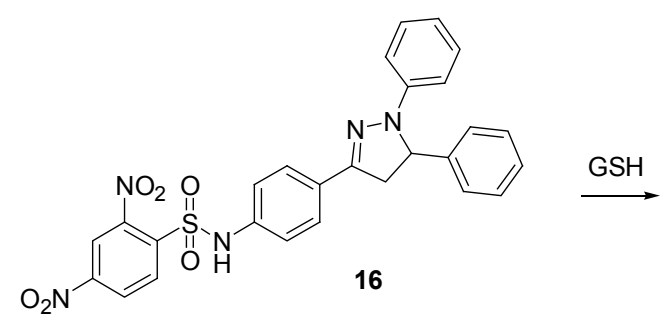<smiles>Nc1ccc(C2=NN(c3ccccc3)C(c3ccccc3)C2)cc1</smiles>

\section{4 基于硫醇参与的二硫键断裂反应.}

$\mathrm{Zhu}$ 等 ${ }^{[62]}$ 报道了基于萗二甲酰亚胺荧光团的含二
硫键的比例荧光探针 17. 在乙醇-PBS $(20 \mathrm{mmol} / \mathrm{L}, \mathrm{pH}=$ 7.4, $V: V=1: 9)$ 的缓冲溶液中, 探针 17 与还原型谷胱 甘肽发生作用，二硫键断裂，在紫外可见吸收光谱中最 大吸收峰红移 $85 \mathrm{~nm}$, 溶液颜色从无色变成翠绿色. 在 苂光图谱中最大发射峰红移 $48 \mathrm{~nm}$, 荧光强度的比值 $\left(F_{533} / F_{485}\right)$ 为 $0.5 \sim 5.7$ (Scheme 1). 对还原型谷胱甘肽的 最低检测限为 $28 \mathrm{~mol} / \mathrm{L}$. 更重要的是, 在 HeLa 细胞中可 以观察到探针 17 的不同的荧光变化.
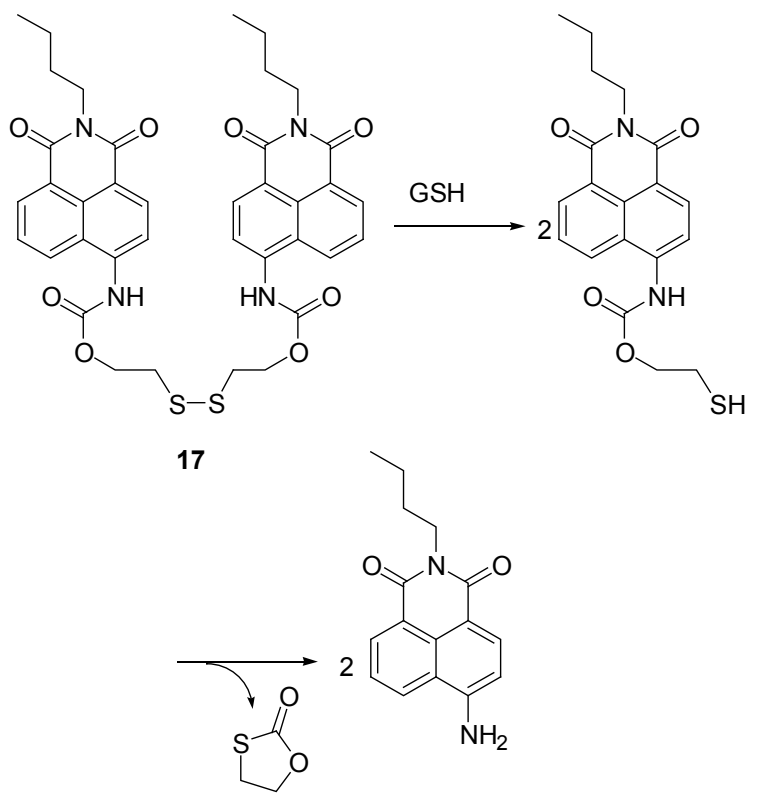

\section{Scheme 1}

$\operatorname{Lim}$ 等 ${ }^{[63]}$ 报道了以 7-氨基香豆素为荧光团，二硫键 为识别基团的比例荧光探针 18 . 在 $0.10 \mathrm{~mol} / \mathrm{L}, \mathrm{pH}=7.4$ 的 HEPES 缓冲溶液中, 探针 $(20 \mathrm{~mol} / \mathrm{L})$ 与还原型谷胱甘 肽 $(20 \mathrm{mmol} / \mathrm{L})$ 作用，随时间延长 $400 \mathrm{~nm}$ 苂光强度降低， $440 \mathrm{~nm}$ 荧光强度增加. 苂光强度比值 $F_{440} / F_{400}$ 由 0.50 增 强到 9.3, 探针与还原型谷胱甘肽作用的二级速率常数 为 $k_{2}=6.5 \times 10^{-3} \mathrm{~L} \cdot \mathrm{mol}^{-1} \cdot \mathrm{S}^{-1}$, 最低检测限为 $6.0 \mathrm{~mol} / \mathrm{L}$, $\mathrm{pH}$ 范围为 $8 \sim 13$. 在文献[64]中有类似探针.

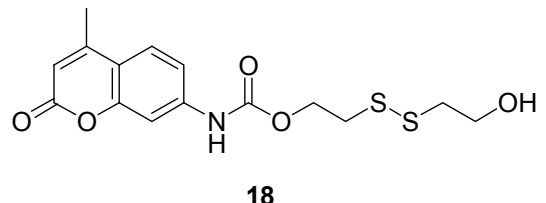

\section{5 基于硫醇与酯的反应}

Yuan 等 ${ }^{[65]}$ 报道的双光子苂光探针 19, 以苯并吡喃 为绿色荧光团，以硫代碳酸酯为识别基团，在含有 $1 \%$ 乙醇的磷酸盐缓冲溶液 $(25 \mathrm{mmol} / \mathrm{L}, \mathrm{pH}$ 7.4) 中与小分子 生物硫醇作用(Eq. 10), $516 \mathrm{~nm}$ 处苂光增强 36 倍. 探针 与还原型谷胱甘肽、半胱氨酸和同型半胱氨酸作用的一 级反应常数分别为 $k=0.05828,0.06082$ 和 0.04421 
$\min ^{-1}$; 探针与还原型谷胱甘肽作用的二级反应常数为 $0.04843 \mathrm{~L} \cdot \mathrm{mol}^{-1} \cdot \mathrm{min}^{-1}$. 探针 19 分别与还原型谷胱甘肽 和半胱氨酸的最低检测限分别为 0.80 和 $0.75 \mathrm{~mol} / \mathrm{L}$. 在 双光子成像实验中, 探针可以检测小白鼠肝脏切片的深 度达到 $180 \mathrm{~m}$. 在小白鼠的活体实验中, 探针主要集中 在小白鼠的肝脏与肾脏中.

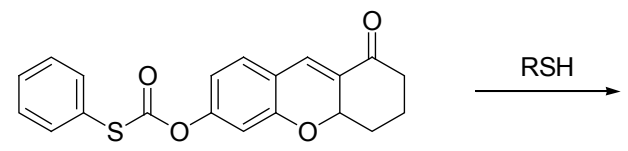

19<smiles>O=C1CCCC2Oc3cc(O)ccc3C=C12</smiles>

\section{6 基于巯基与硒氮键作用.}

Wang 等 ${ }^{[66]}$ 报道了灵敏的近红外苂光硫醇探针 20 和 21 (Eqs. 11, 12). 由于在识别基团和苂光基团之间存 在的供电激发光诱导电子转移(d-PET), 探针 $\mathbf{2 0}$ 和 21 在 PBS 缓冲溶液 (15 mmol/L, $\mathrm{pH} 7.4$ )中溶解 $3 \mathrm{~min}$ 后, 在 $635 \mathrm{~nm}$ 激发时, $750 \mathrm{~nm}$ 处有微弱的荧光. 在探针 20 和 21 溶液中加入谷胱甘肽后, 在 $750 \mathrm{~nm}$ 处的发射峰表现 出显着的苂光增强作用. 此外, 探针 20 和 21 的苂光强 度与 $0 \sim 5 \mathrm{~mol} / \mathrm{L}$ 范围内的半胱氨酸成正比. 共聚焦显微 镜成像证实这两种化合物都可用于检测 RAW 264.7 细 胞和新鲜的大鼠肝组织中的硫醇.

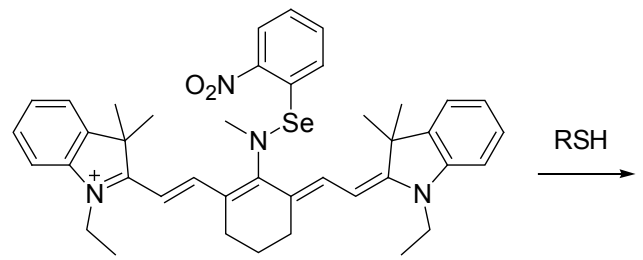

20

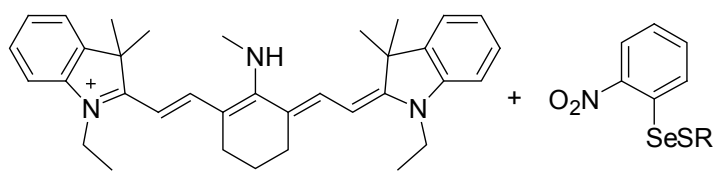

\section{7 基于俆基与呋喃环作用.}

Zhai 等 ${ }^{[67]}$ 报道了以 BODIPY 为荧光基团, 呋喃环为 识别基团的比例荧光探针 22. 在二甲基亚砜与磷酸盐 缓冲溶液中 $(V: V=1: 4,20 \mathrm{mmol} / \mathrm{L}, \mathrm{pH}=7.4)$, 虽然探 针对小分子生物硫醇都有响应, 由于还原谷胱甘肽在生 物体中的含量远远大于半胱氨酸的含量, 并且在荧光光 谱中与还原型谷胱甘肽作用后的荧光强度强于与半胱 氨酸作用的强度, 所以在生物体中探针主要是对还原型

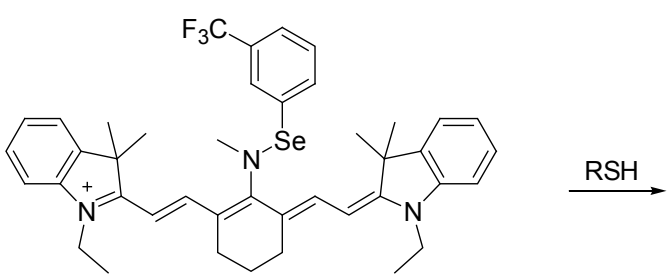

21<smiles>[R][SH](=O)c1cccc(C(F)(F)F)c1</smiles>

谷胱甘肽响应(Scheme 2). 探针 22 与还原型谷胱甘肽作 用后最大发射由 $585 \mathrm{~nm}$ 蓝移到 $522 \mathrm{~nm}$. 动力学常数 $k^{\prime}=2.5 \mathrm{~s}^{-1}$. 在正常条件下探针 22 与还原型谷胱甘肽作 用, 溶液由红色变为绿色, 当缓冲溶液中的 DMSO 增加 到 $50 \%$ 时, 溶液颜色由红色变成橙色然后再变为绿色. 探针用于 $3 \mathrm{~T} 3$ 细胞进行成像实验, 在正常细胞中加入还 原型谷胱甘肽引发剂硫辛酸, $F_{\text {green }} / F_{\text {red }}$ 比值由 1.61 变到 2.59 , 而加入生物硫醇清除剂 $N$-甲基马来酰亚胺的细 胞, 其比值为 0.14 . 探针用于肝脏肿瘤组织, 相对于正 常的肝脏组织, 在肝脏肿瘤组织中探针 22 绿光强, 红光 弱.
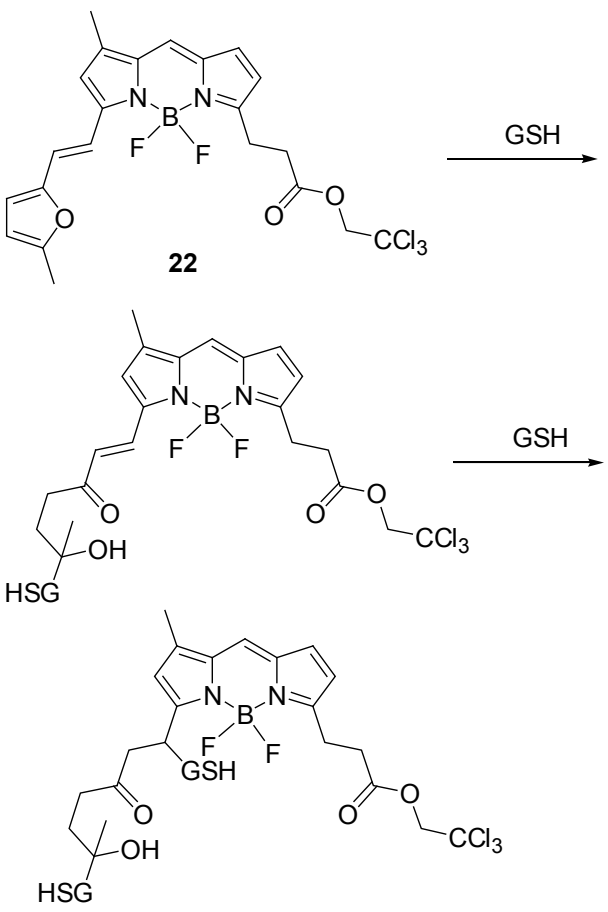

Scheme 2

\section{8 統基与碳氮双键发生亲核加成反应.}

Das 等 ${ }^{[68]}$ 报道了以喹啉为荧光基团的淬灭型荧光探 针 23, 24 (Scheme 3). 在二甲基亚砜与 HEPES 缓冲溶液 
$(50 \mathrm{mmol} / \mathrm{L}, V: V=7: 3, \mathrm{pH}$ 7.4)混合溶液中, 探针 23 的紫外最大吸收峰在 $296 \mathrm{~nm}$, 摩尔消光系数为 $6.44 \times$ $10^{3} \mathrm{dm}^{3} \cdot \mathrm{mol}^{-1} \cdot \mathrm{cm}^{-1}$, 而在 $365 \mathrm{~nm}$ 吸收峰的摩尔消光系 数为 $3.85 \times 10^{3} \mathrm{dm}^{3} \cdot \mathrm{mol}^{-1} \cdot \mathrm{cm}^{-1}$, 当用 $296 \mathrm{~nm}$ 激发探针 23 时, 最大发射峰波长为 $342 \mathrm{~nm}$. 在探针 23 溶液中加 入还原型谷胱甘肽后, 随着时间的延长, $365 \mathrm{~nm}$ 紫外吸 收峰逐渐消失, 在荧光光谱中 $342 \mathrm{~nm}$ 处的荧光强度逐 渐淬灭, 探针 23 与还原型谷脱甘肽反应完全的时间为

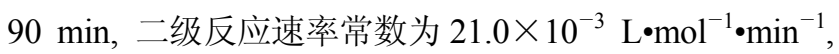
半衰期寿命为 $28.7 \mathrm{~min}$. 探针 $\mathbf{2 4}$ 与还原型谷胱甘肽反应 速率比探针 23 的反应速率慢, 主要是因为探针 23 结构 中存在分子内氢键作用, 增强了席夫碱中碳原子的正电 性, 有利于亲核试剂颈基的进攻. 探针 23, 24 都可以与 硫醇小分子化合物作用. 苂光强度与还原型谷胱甘肽在 $(1 \sim 36) \times 10^{-4} \mathrm{~mol} / \mathrm{L}$ 范围内成很好的线性关系. 探针 23 不仅用于活体 HeLa 细胞而且用于人体血浆中检测生物 硫醇.
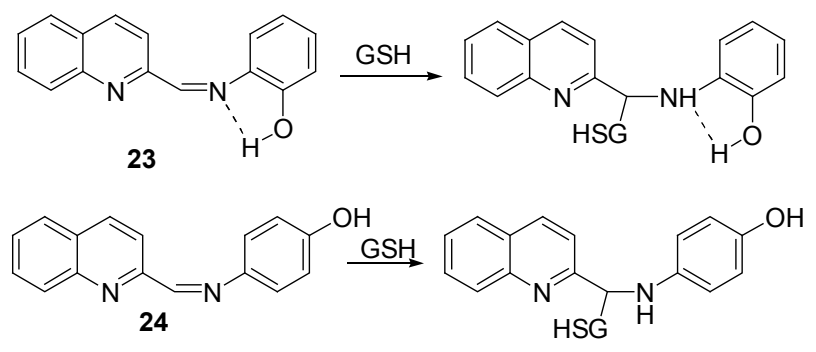

Scheme 3

\section{9 統基还原偶氮化合物}

小分子硫醇中的巯基不仅具有亲核性，还具有还原 性, 可以与氧化剂发生氧化还原反应. 已报道的小分子 生物硫醇荧光探针大多利用了硫醇分子中統基的亲核 性, 而利用颈基的还原性检测小分子生物硫醇的文献报 道尚不多见.

$\mathrm{Li}$ 等 ${ }^{[69]}$ 报道了金属铱络合物为荧光团, 偶氮化合 物为检测基团的磷光探针 25 (Eq. 13). 在乙腈与 HEPES 缓冲溶液 $(10 \mathrm{mmol} / \mathrm{L}, \mathrm{pH} 7.5, V: V=1: 1)$ 的混合溶液 中, $2.5 \mathrm{~mol} / \mathrm{L}$ 的探针 25 与 $25 \mathrm{~mol} / \mathrm{L}$ 的氨基酸作用, 探针 只对含有颈基的半胱氨酸、同型半胱氨酸和还原型谷胱 甘肽有响应, 磷光最大发射峰在 $564 \mathrm{~nm}$ 处. 在 $\mathrm{pH}=4 \sim$ 10 范围内探针对小分子生物硫醇都有作用. 逐渐增大 半胱氨酸、同型半胱氨酸和还原型谷胱甘肽的浓度, 磷 光强度分别增强 30,38 和 35 倍; 最低检测限分别为 $3.69 \times 10^{-8}, 6.76 \times 10^{-8}$ 和 $2.40 \times 10^{-8} \mathrm{~mol} / \mathrm{L}$. ES-MS 图谱 证明了探针 25 中的偶氮基团与硫醇作用, 被还原成了 两个阴离子. 探针 25 成功用于 HeLa 细胞成像.

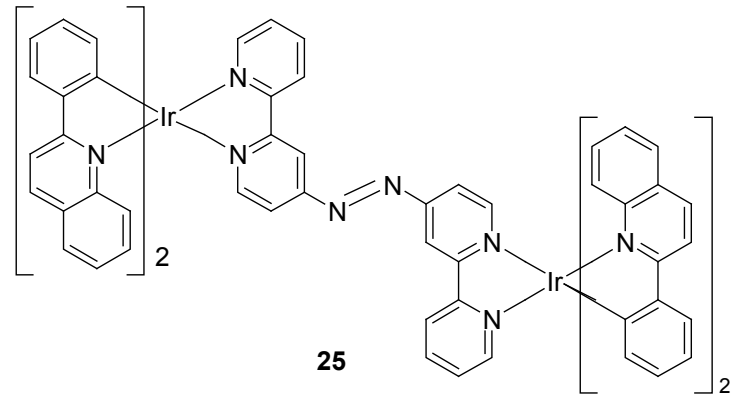

thiol

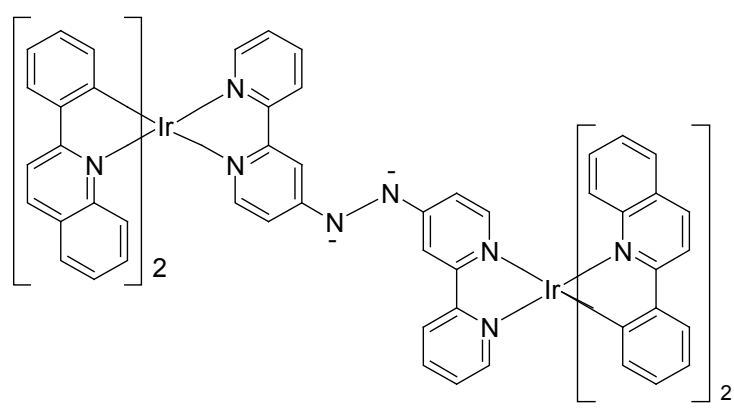

(13)

\subsection{0 小分子生物硫醇中巯基与金属络合物作用}

小分子生物硫醇中的䘪基除了具有亲核性、还原性 之外, 还具有很强地与过渡金属发生配位的能力, 夺取 苂光配体与金属配合物中的金属, 释放出荧光化合物, 以检测小分子生物硫醇化合物.

Wang 等 ${ }^{[70]}$ 合成了苂光素席夫碱探针 $\mathbf{2 6}$ 与 $\mathrm{Cu}^{2+}$ 的 络合物, 可以在水溶液中检测生物硫醇(Scheme 4). 在 含有 $1 \%$ 乙腈的 HEPES 缓冲溶液 $(0.02 \mathrm{~mol} / \mathrm{L}, \mathrm{pH} 7.4$, 中, 探针 26- $\mathrm{Cu}^{2+}$ 与半胱氨酸作用, 在 $515 \mathrm{~nm}$ 处苂光增 强, $\mathrm{pH}$ 作用范围为 $5 \sim 10$. 在浓度为 $2 \mathrm{~mol} / \mathrm{L}$ 的探针中逐 渐加入半胱氨酸, 半胱氨酸浓度在 $0 \sim 20 \mathrm{~mol} / \mathrm{L}$ 范围内, 具有很好的线性关系, 最低检测限为 $9 \mathrm{~mol} / \mathrm{L}$. 作用机 理可能是半胱氨酸夺取络合物中的 $\mathrm{Cu}^{2+}$, 然后席夫碱 水解生成醛而发出苂光. 他们把探针 $\mathbf{2 6}-\mathrm{Cu}^{2+}$ 用于了人 体乳腺癌细胞中进行了生物成像实验.

\section{2 利用小分子生物硫醇中颈基、氨基双官能团 检测生物硫醇}

利用小分子生物硫醇中颈基与氨基的亲核性, 设计 合成小分子生物硫醇探针, 提高对半胱氨酸、同型半胱 氨酸和还原型谷胱甘肽的选择性.

\section{1 基于半胱氨酸或同型半胱氨酸的共轭加成环化反 应的荧光探针}

已有报道对半胱氨酸和同型半胱氨酸具有很好选 择性探针, 但是利用同一个探针, 区分半胱氨酸与同型 


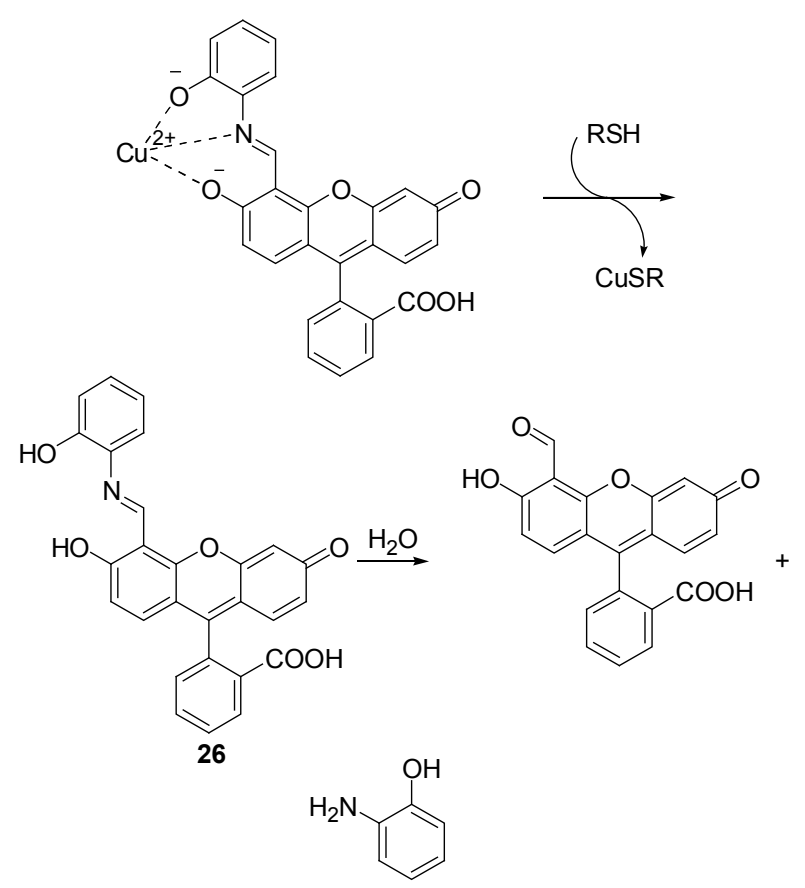

Scheme 4

半胱氨酸还是很困难的. 这是因为二者的结构类似, 二 者之间唯一的区别在于同型半胱氨酸比半胱氨酸多一 个亚甲基.

在 2011 年, Yang 等 ${ }^{[71]}$ 报道了苯并噻唑衍生物探针 27, 可以在中性溶液中选择半胱氨酸与同型半胱氨酸 (Scheme 5). 这个方法包括先共轭加成生成硫醚, 然后 再环化生成 2-(2'-着基-3'-甲氧基苯基)苯并噻唑(HMBT). 环形成的动力学差异, 允许光谱或动力学模式来区分半 胱氨酸或者同型半胱氨酸. 还原型谷胱甘肽只能与探针 发生迈克尔加成反应, 不能发生氨解成环释放出羟基, 发生异构使得荧光图谱发生红移. 因此探针 27 可以利 用作用时间区分半胱氨酸、同型半胱氨酸与还原型谷胱 甘肽. 此外, 探针 27 成功地应用于检测稀释了的脱蛋白 人血浆中的半胱氨酸和同型半胱氨酸.
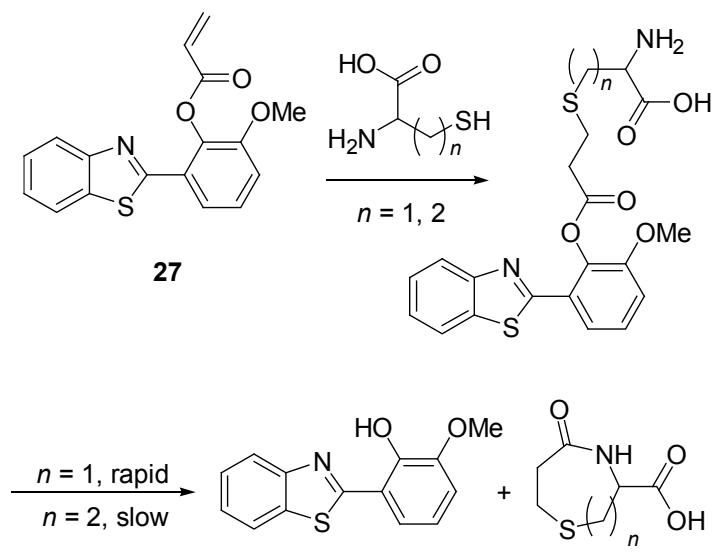

Scheme 5
Guo 等 ${ }^{[72]}$ 以川菁染料为荧光基团设计合成了近红 外苂光比例探针 $\mathbf{2 8}$, 可以高效地把半胱氨酸与同型半 胱氨酸和谷胱甘肽进行区分(Eq. 14). 该探针以丙烯酸 酯为硫醇的识别基团，当加入半胱氨酸以后，探针 $\mathbf{2 8}$ 先 加成然后再环化生成羟基川菁, 然后异构化生成化合物 28'. 相应地, 探针 28 在溶液中表现出明显的紫外可见 吸收和荧光发射光谱的变化(紫外可见吸收光谱从 770 $\mathrm{nm}$ 蓝移到 $515 \mathrm{~nm}$, 荧光发射光谱从 $780 \mathrm{~nm}$ 移到 570 $\mathrm{nm})$. 探针 $\mathbf{2 8}$ 对半胱氨酸的选择性响应归功于探针 $\mathbf{2 8}$ 与硫醇化合物的加成环化的反应速率不同. 探针 28 对 半胱氨酸的响应被进一步应用到活细胞内生物成像. 探 针 28 在对用无糖 DMEM 改良的 Eagle 培养液培养的 MCF-7 细胞作用时, 在 $590 \mathrm{~nm}$ 处出现较强的苂光而在 近红外区域 $(760 \sim 855 \mathrm{~nm}$ ) 的苂光急剧减弱. 众所周知, 如果在 MCF-7 细胞中没有葡萄糖，则细胞内的半胱氨 酸浓度明显增加. 当使用两种不同的成像信号通道扫描 细胞时, 在细胞内探针 28 与半胱氨酸作用显示了很好 的比例成像.

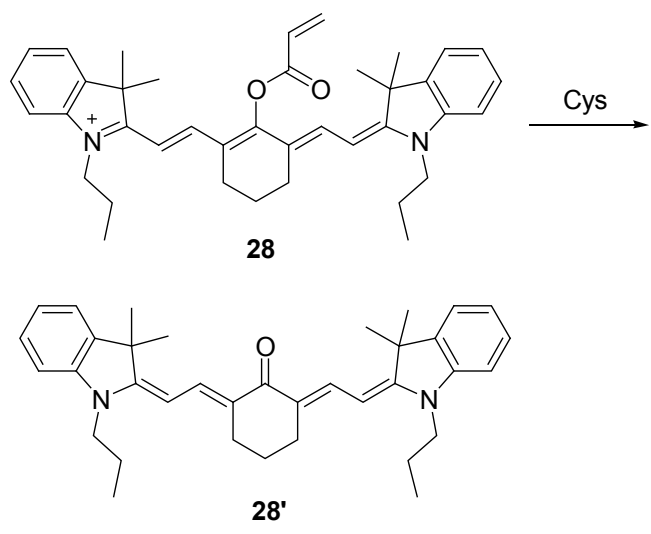

Xiong 等 ${ }^{[73]}$ 报道了近红外探针 29 , 探针 29 以苯并 吡喃类化合物为荧光基团，丙烯酸酯为识别基团，可以 快速地检测半胱氨酸 (Scheme 6). 在浓度为 $40 \mathrm{mmol} / \mathrm{L}$, $\mathrm{pH}=7.37$ 的 HEPES 缓冲溶液中, $5 \mathrm{~mol} / \mathrm{L}$ 的探针 29 与 34 倍半胱氨酸、同型半胱氨酸和还原型谷胱甘肽作用, 在 $10 \mathrm{~min}$ 之内探针可以与半胱氨酸作用完全，而同型半 胱氨酸与还原型谷胱甘肽作用完全最少需要 $1 \mathrm{~h}$. 在探 针溶液中加入半胱氨酸后, 在 $10 \mathrm{~min}$ 之内苂光强度在 $650 \mathrm{~nm}$ 处大约增强 20 倍. 当半胱氨酸浓度在 $0 \sim 40$ $\mathrm{mol} / \mathrm{L}$ 之间时，苂光强度与半胱氨酸成很好的线性关系， 最低检测限为 $0.0309 \mathrm{~mol} / \mathrm{L}$. 在紫外可见吸收光谱中, 随着半胱氨酸浓度的增大，在 474 和 $520 \mathrm{~nm}$ 处的吸收峰 逐渐减弱，在 576 和 $625 \mathrm{~nm}$ 处出现新的吸收峰，等消光 点出现在 $525 \mathrm{~nm}$ 处. 作者把探针 29 用于了 MCF-7 细胞 中进行了细胞成像, 然后又把 $0,10,20$ 和 $50 \mathrm{~mol} / \mathrm{L}$ 浓度 的探针注射活体老鼠中，成功地进行了活体动物成像实 
验.<smiles>C=CC(=O)Oc1cc2c(cc1Cl)C(c1ccccc1C(=O)O)=C1CCCC(C=C(C#N)C#N)=C1O2</smiles><smiles>CCCC1=C(C=C(C#N)C#N)Oc2cc(OC(=O)CCSCC(N)C(=O)O)c(Cl)cc2C1=C1OC(=O)c2ccccc21</smiles><smiles>N#CC(C#N)=CC1=C2CCCC(=C(c3ccccc3C(=O)O)c3cc(Cl)c(O)cc3O2)C1=O</smiles>

Scheme 6

Wang 等 ${ }^{[74]}$ 报道了以 $1,3,5$-三芳基吡唑啉为荧光团 的荧光探针 30, 检测还原型谷胱甘肽(Eq. 15). 探针 30 在室温条件、乙醇-PBS $(10 \mathrm{mmol} / \mathrm{L} \mathrm{pH}=7.4, V: V=3$ : 7)的缓冲溶液中加入还原型谷胱甘肽或者半胱氨酸后, $474 \mathrm{~nm}$ 荧光强度增强, 随着时间的变化, 探针 $\mathbf{3 0}$ 与半 胱氨酸发生迈克尔加成产物会发生分子内氨解生成非 苂光化合物从而荧光淬灭; 然而探针 $\mathbf{3 0}$ 与还原型谷胱 甘肽的加成产物 $30^{\prime}$ 中氨基由于位置原因不发生分子内 氨解反应, 因此苂光现象保持不变. 还原型谷胱甘肽在 浓度为 $0 \sim 10 \mathrm{~mol} / \mathrm{L}$ 范围内, 浓度与苂光强度成线性关 系, 最小检测浓度为 $82 \mathrm{nmol} / \mathrm{L} . \mathrm{pH}$ 值范围广为 $6.5 \sim$ 10.5 , 作用时间为 $3 \mathrm{~h}$. 共聚焦显微镜成像证实探针 $\mathbf{3 0}$ 可用于检测 HeLa 细胞中的还原型谷胱甘肽.

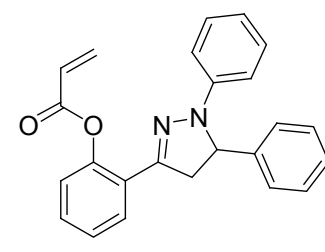

30

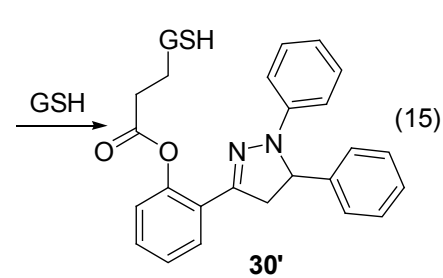

$30^{\prime}$
Dai 等 ${ }^{[75]}$ 合成香豆素荧光基团半胱氨酸探针 31 (Eq. 16). 在室温条件、乙醇-HEPES $(10 \mathrm{mmol} / \mathrm{L}, \mathrm{pH}=7.4$, $V: V=2: 3$ )的缓冲溶液中加入半胱氨酸后, 体系颜色 由无色变为黄色, 最大紫外可见吸收峰出现在 $424 \mathrm{~nm}$,
最大发射波长在 $456 \mathrm{~nm}$, 探针 31 具有很强的抗干扰能 力. 半胱氨酸的浓度是探针浓度的 6 equiv. 时, 在 $40 \mathrm{~min}$ 内苂光探针 31 与半胱氨酸就可以作用完全, 与文献相 比具有半胱氨酸浓度低, 作用时间短的优点. 在测试体 系中, 荧光强度随着半胱氨酸浓度的增加逐渐增强, 在 0 40 mol/L 范围, 成线性关系, 最低检测限为 0.657 $\mathrm{mol} / \mathrm{L}$, 相关系数 $R=0.9970$. 探针 31 可以检测 $\mathrm{HeLa}$ 细 胞与小牛血清中的半胱氨酸.

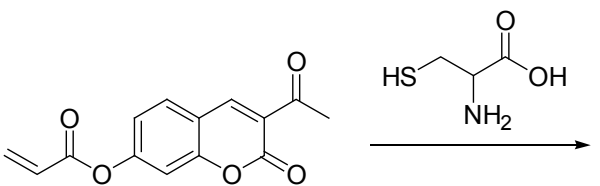

31<smiles>CC(=O)c1cc2ccc(O)cc2oc1=O</smiles>

\section{2 基于半胱氨酸或同型半胱氨酸与醛成环反应的苂 光探针}

醛基官能团可以与半胱氨酸中的氨基发生缩合反 应，然后与統基再发生加成反应，生成噻唑啉环，并且 与同型半胱氨酸相比, 成环的碳数不同, 因此存在着反 应速率的差异. 由于氨基与颈基距离较远还原型谷胱甘 肽难以发生类似反应. 这类反应主要是半胱氨酸荧光探 针.

Wang 等 ${ }^{[76]}$ 报道了基于荟二甲酰亚胺为苂光基团的 乙二醛腙探针 32 (Eq. 17). 由于探针 32 与半胱氨酸或同 型半胱氨酸的作用, 生成噻唑啉形成分子内氢键从而抑 制了 $\mathrm{C}=\mathrm{N}$ 双键发生异构化而引起的荧光淬灭, 成为荧 光增强型探针. 探针 32 与半胱氨酸和同型半胱氨酸作 用生成噻唑啉环，环上 $\mathrm{N}$ 原子上的孤对电子形成了分子 内氢键阻碍了 $\mathrm{C}=\mathrm{N}$ 双键的异构化, 从而探针 32 在二甲 基亚砜溶液中在 524 纳米处苂光增强. 此外, 在 DMSO-HEPES 缓冲液中 $(100 \mathrm{mmol} / \mathrm{L}, \mathrm{pH} 7.4, V: V=$ $1: 1)$ 探针 32 苂光增强的剂量依赖性, 与半胱氨酸和同 型半胼氨酸在 $0 \sim 500 \mathrm{~mol} / \mathrm{L}$ 表现出良好的线性关系, 成 功地应用到生物成像中检测活细胞内半胱氨酸或同型 半胱氨酸.

Kong 等 ${ }^{[77]}$ 报道的近红外荧光探针 $\mathbf{3 3}$, 以花青染料 为荧光基团, 醛基为识别基团, 可以有效地检测半胱氨 酸与同型半胱氨酸(Eq. 18). 在 $37{ }^{\circ} \mathrm{C}$ 条件下, $\mathrm{pH}=7.4$ 浓 度为 $40 \mathrm{mmol} / \mathrm{L}$ 的磷酸盐缓冲溶液中, 在探针 33 中逐渐 加入半胱氨酸, 在 $778 \mathrm{~nm}$ 处的荧光强度逐渐增强, 荧光 量子产率由 0.036 增强到 0.089 . 迄今文献报道的生物硫 
<smiles>CCCCN1C(=O)c2cccc3c(NN=CC=O)ccc(c23)C1=O</smiles><smiles></smiles>

醇小分子苂光探针中，探针 33 检测限最低，为 7.9 $\mathrm{nmol} / \mathrm{L}$. 作者用质谱验证了探针 33 与半胱氨酸作用生 成噻唑啉的反应机理, 并且把探针成功地用于 HepG2 细胞成像, 具有很好的光化学稳定性.
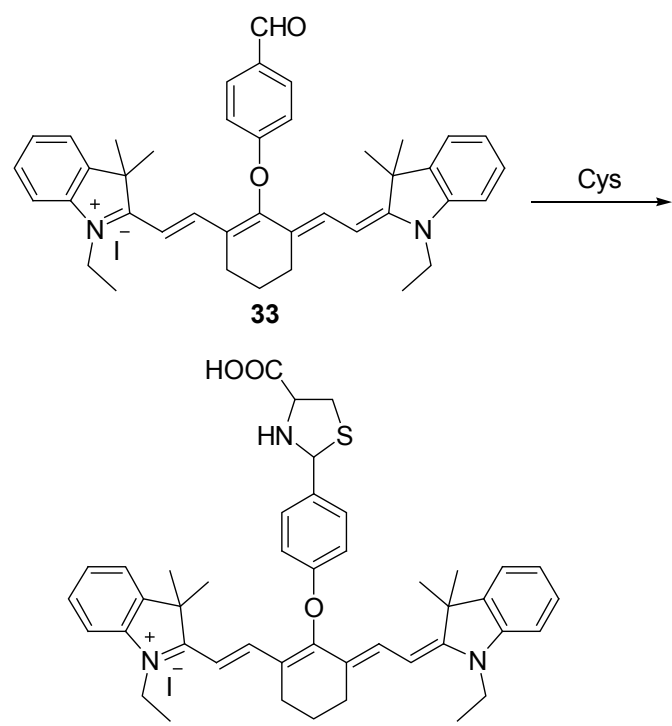

\section{3 基于半胱氨酸与醛基缩合再与双键加成成环反应 的荧光探针}

Yuan 等 ${ }^{[78]}$ 报道了以香豆素为苂光基团的增强型比 例苂光探针 34. 在乙腈与 $\mathrm{PBS}$ 缓冲溶液 $(V: V=9: 1$, $\mathrm{pH}$ 7.4)混合溶液中, 探针 34 的最大发射峰在 $557 \mathrm{~nm}$ 处, 量子产率为 0.12 , 当加入半胱氨酸后, $557 \mathrm{~nm}$ 处的苂光 强度逐渐减弱, $487 \mathrm{~nm}$ 处苂光强度突然增强, 量子产率 为 0.25 , 发射波长蓝移 $70 \mathrm{~nm}$. 苂光强度比值 $I_{487} / I_{557}$ 由 最初的 0.054 增大到 6.24, 增大了 115 倍, 最低检测限为 $7.5 \times 10^{-7} \mathrm{~mol} / \mathrm{L}(S / N=3)$. 探针 34 与半胱氨酸、同型半 胱氨酸和还原型谷胱甘肽反应的二级反应速率分别为

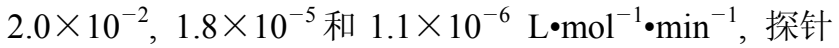
34 与半胱氨酸的反应速率远远大于同型半胱氨酸和还 原型谷胱甘肽, 因此探针 34 可以有效地区分半胱氨酸 与同型半胱氨酸、还原型谷胱甘肽 (Scheme 7). 在 MCF-7 细胞内探针 34 与半胱氨酸作用成功的显示了很
好的比例成像.

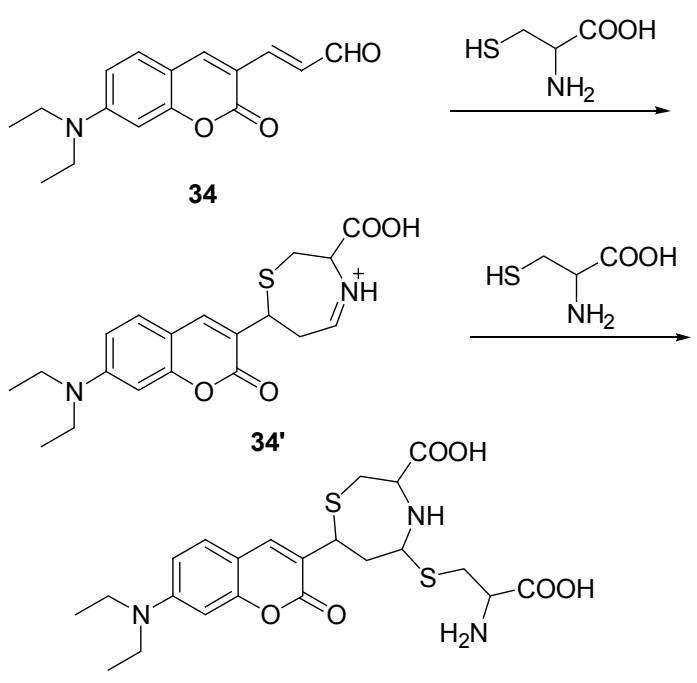

Scheme 7

\section{4 基于半胱氨酸发生分子内亲核取代反应的荧光探 针}

$\mathrm{Ma}$ 等 $^{[79]}$ 报道了以 4-硝基-1,8-萗二甲酸酐为荧光基 团的增强型荧光探针 35. 探针 35 溶解在 $N, N$-二甲基甲 酰胺溶液中为无色无荧光溶液. 探针 35 的紫外可见最 大吸收峰在 $345 \mathrm{~nm}$, 加入半胱氨酸 $0 \sim 15 \mathrm{~min}$ 之内, 在 $345 \mathrm{~nm}$ 处的最大吸收峰减弱, 在 $390 \mathrm{~nm}$ 出现新的吸收 峰, 等消光点在 $365 \mathrm{~nm} ; 15 \mathrm{~min}$ 以后 $390 \mathrm{~nm}$ 的最大吸收 峰减弱, $435 \mathrm{~nm}$ 出现新的最大吸收峰, 等消光点在 410 $\mathrm{nm}$, 溶液颜色由无色变为黄色(Scheme 8). 在 $0 \sim 15 \mathrm{~min}$ 之内用 $390 \mathrm{~nm}$ 波长激发, 最大发射峰由 $450 \mathrm{~nm}$ 红移到 $490 \mathrm{~nm}$; 在 $15 \mathrm{~min}$ 以后用 $435 \mathrm{~nm}$ 波长激发, 最大发射 峰出现在 $530 \mathrm{~nm}$. 半胱氨酸中的颈基可以与 4-硝基-1,8荟二甲酸䣶发生芳香亲核反应，生成的硫醚化合物又能 发生分子内的氨基亲核取代反应，两种物质的最大吸收 峰与最大发射波长不同，因此导致上述现象. 在 $0 \sim 30$ $\mathrm{mol} / \mathrm{L}$ 范围内半胱氨酸浓度与荧光强度成线性关系, 最 小检测浓度为 $3 \times 10^{-7} \mathrm{~mol} / \mathrm{L}(S / N=3)$.<smiles>O=C1OC(=O)c2ccc([N+](=O)[O-])c3cccc1c23</smiles><smiles>NC(CS)C(=O)OCC(=O)O</smiles><smiles>NC(CSc1ccc2c3c(cccc13)C(=O)OC2=O)C(=O)O</smiles> 


\section{5 基于亲核取代一加成成环反应的荧光探针}

$\mathrm{Liu}$ 等 ${ }^{[80]}$ 报道了以香豆素为荧光基团的增强型苂光 探针 36. 在 PB 缓冲溶液 $(10 \mathrm{mmol} / \mathrm{L}, \mathrm{pH} 7.4,1 \mathrm{mmol} / \mathrm{L}$ CTAB)溶液中, 探针 36 的紫外可见最大吸收峰在 535 $\mathrm{nm}$, 加入半胱氨酸后, 在 $535 \mathrm{~nm}$ 处的最大吸收峰减弱; 在 $360 \mathrm{~nm}$ 出现新的吸收峰, 然而加入谷胱甘肽后, 最大 吸收峰红移到 $558 \mathrm{~nm}$, 然后减弱, 在 $450 \mathrm{~nm}$ 出现新的 吸收峰. 在紫外可见光谱中出现新的吸收峰, 说明探针 36 与半胱氨酸和还原型谷胱甘肽作用的产物不同. 半 胱氨酸的颈基先与探针 36 中的卤素发生亲核取代反应. 然后再分子内亲核反应, 释放出来的巯基在于双键发生 加成反应; 谷胱甘肽发生亲核取代反应以后, 由于氨基 位置原因直接与苯并噻唑中的碳氮双键发生了加成反 应，同型半胱氨酸只能发生亲核取代反应. 探针 36 与 Cys, Hcy 和 GSH 反应后的产物不同. 苂光图谱中的最 大发射峰也不同, 探针 36 中加入半胱氨酸时, 用 360 $\mathrm{nm}$ 激发, 最大发射峰在 $420 \mathrm{~nm}$; 加入谷胱甘肽时, 用 $450 \mathrm{~nm}$ 激发, 最大发射峰在 $512 \mathrm{~nm}$; 同型半胱氨酸在 以上两个波长激发时, 苂光发射峰都很弱. 探针 $\mathbf{3 6}$ 对半 胱氨酸和还原型谷胱甘肽的最低检测限分别为 $0.4,0.05$
mol/L (Scheme 9). 成功地把探针 36 用于了 COS-7 细胞 中进行了生物成像实验, 并且分别增大细胞中半胱氨酸 或者还原型谷胱甘肽的浓度，细胞成像的相应颜色也随 之变化.

\section{3 结束语}

近几年来，荧光探针在生物硫醇检测和应用方面取 得了令人振奋的进展. 根据硫醇探针的反应机制, 本文 将荧光探针和比色传感器进行了分类与概述: 小分子生 物硫醇中䘪基与探针反应; 利用小分子生物硫醇中䘪 基、氨基双官能团检测生物硫醇. 尽管有大量的文献报 道了生物硫醇荧光探针, 但是仍然有众多的工作需要探 索研究，可以同时区别半胱氨酸、同型半胱氨酸和还原 型谷胱甘肽的检测基团的发现，或者是可以对其中某一 个硫醇化合物具有专一性识别的检测基团; 开发具有新 的近红外或者是双光子荧光基团的生物硫醇探针, 以及 含有这些荧光基团的比率探针; 具有靶向特定器官或细 胞器药物传递系统(DDS)的生物硫醇荧光探针. 在众多 科学工作者的努力下, 荧光探针在检测生物硫醇方面将 获得进一步发展.<smiles>CCNc1ccc2c(Cl)c(/C=C/c3sc4ccccc4[n+]3C)c(=O)oc2c1</smiles><smiles>CCN(CC)c1ccc2c(NC(CS)C(=O)O)c(/C=C/c3sc4ccccc4[n+]3C)c(=O)oc2c1</smiles>

36<smiles>CCNc1ccc2c([Se])c(/C=C/c3sc4ccccc4[n+]3C)c(=O)oc2c1</smiles><smiles>CCN(CC)c1ccc2c(SCC(NC(=O)CCC(=O)O)C(=O)NCC(=O)O)c(/C=C/[C@@]3(N(C)CC)Sc4ccccc4N3C)c(=O)oc2c1</smiles><smiles>CCNc1ccc2c(NC(C(=O)O)C(=O)O)c(C(Cc3sc4ccccc4[n+]3C)SSCC)c(=O)oc2c1</smiles>

Scheme 9

\section{References}

[1] Zhang, S. Y.; Ong, C. N.; Shen, H. M. Cancer Lett. 2004, 208, 143.

[2] Townsend, D. M.; Tew, K. D.; Tapiero, H. Biomed. Pharmacother. 2003, 57, 145.

[3] Kemp, M.; Go, Y. M.; Jones, D. P. Free Radical Biol. Med. 2008, 44, 921.

[4] Reddie, K. G.; Carroll, K. S. Curr. Opin. Chem. Biol. 2008, 12, 746.

[5] Weerapana, E.; Wang, C.; Simon, G. M.; Richter, F.; Khare, S.; Dillon, M. B. D.; Bachovchin, D. A.; Mowen, K.; Baker, D.; Cravatt, B. F. Nature 2010, 468, 790.

[6] Lee, M. H.; Han, J. H.; Kwon, P. S.; Bhuniya, S.; Kim, J. Y.; Sessler, J. L.; Kang, C.; Kim, J. S. J. Am. Chem. Soc. 2012, 134, 1316.

[7] Chen, Z.; Wang, Z.; Chen, J.; Chen, X. Biosens. Bioelectron. 2012,
$38,202$.

[8] Wu, S.; Lan, X.; Huang, F.; Luo, Z.; Ju, H.; Meng, C.; Duan, C. Biosens. Bioelectron. 2012, 32, 293.

[9] Weerapana, E.; Wang, C.; Simon, G. M.; Richter, F.; Khare, S.; Dillon, M. B. D.; Bachovchin, D. A.; Mowen, K.; Baker, D.; Cravatt, B. F. Nature 2010, 468, 790.

[10] Shahrokhian, S. Anal. Chem. 2001, 73, 5972.

[11] Elshorbagy, A. K.; Kozich, V.; Smith, A. D.; Refsum, H. Curr. Opin. Clin. Nutr. Metab. Care 2012, 15, 49.

[12] Cankurtaran, M.; Yesil, Y.; Kuyumcu, M. E.; Oztürk, Z. A.; Yavuz, B. B.; Halil, M.; Ulger, Z.; Cankurtaran, E. S.; Arıogul, S. J. Alzheimer's Dis. 2013, 33, 1051.

[13] Seshadri, S.; Beiser, A.; Selhub, J.; Jacques, P. F.; Rosenberg, I. H.; D'Agostino, R. B.; Wilson, P. W. F.; Wolf, P. A. N. Engl. J. Med. 2002, 346, 476. 
[14] Hankeya, G. J.; Eikelboom, J. W. Curr. Opin. Neurol. 2001, 14, 95.

[15] Morgenstern, I.; Raijmakers, M. T. M.; Peters, W. H. M.; Hoensch, H.; Kirch, W. Dig. Dis. Sci. 2003, 48, 2083.

[16] Dalton, T. P.; Shertzer, H. G.; Puga, A. Annu. Rev. Pharmacol. Toxicol. 1999, 39, 67.

[17] Vreuls, C. P. H.; Damink, S. W. M. O.; Koek, G. H.; Winstanley, A.; Wisse, E.; Cloots, R. H. E.; Broek, M. A. J.; Dejong, C. H. C.; Bosman, F. T.; Driessen, A. Br. J. Cancer 2013, 108, 676.

[18] Caccamo, A.; Medina, D. X.; Oddo, S. J. Neurosci. 2013, 33, 906.

[19] Watanabe, Y.; Watanabe, K.; Kobayashi, T.; Saito, Y.; Fujioka, D.; Nakamura, T.; Obata, J.; Kawabata, K.; Mishina, H.; Kugiyama, K. Cardiovasc. Res. 2013, 97, 282.

[20] Liu, W. Y.; Li, H. Y.; Zhao, B. X.; Miao, J. Y. Analyst 2012, 137, 3466.

[21] Yuan, L.; Lin, W.; Chen, B.; Xie, Y. Org. Lett. 2012, 14, 432.

[22] Liu, W. Y.; Shen, S. L.; Li, H. Y.; Miao, J. Y.; Zhao, B. X. Anal. Chim. Acta 2013, 791, 65.

[23] Zhang, G.; Wen, Y.; Guo, C.; Xu, J.; Lu, B.; Duan, X.; He, H.; Yang, J. Anal. Chim. Acta 2013, 805, 87.

[24] Xu, Y.; Jiang, Z.; Xiao, Y.; Zhang, T. T.; Miao, J. Y.; Zhao, B. X. Anal. Chim. Acta 2014 807, 126.

[25] Li, C. Y.; Kong, X. F.; Li, Y. F.; Weng, C.; Tang, J. L.; Liu, D.; Zhu, W. G. Anal. Chim. Acta 2014, 824, 71.

[26] Zhang, Y.; Wang, Q.; Li, W.; Zhang, J.; Xia, M.; Chen, S.; Chen, X. Chin. J. Org. Chem. 2014, 34, 403 (in Chinese).

(张勇, 王强, 李伟, 张静, 夏明辉, 陈世明, 陈雪梅, 有机化学, 2014, 34, 403.)

[27] Meng, W.; Yang, M.; Chen, Z.; Li, S.; Yang, B. Chin. J. Org. Chem. 2014, 34, 398 (in Chinese). (孟文斐, 杨美盼, 成昭, 李少妮, 杨秉勤, 有机化学, 2014, 34, 398.)

[28] Su, W.; Tong, J.; Yang, B. Chin. J. Org. Chem. 2013, 33, 982 (in Chinese).

(苏文崎, 童杰, 杨秉勤, 有机化学, 2013, 33, 982.)

[29] Chen, J.; Tian, Y.; Xiang, Q.; Xiong, J.; Zhang, L.; Li, Y. Chin. J. Org. Chem. 2013, 33, 2210 (in Chinese).

(陈稼轩, 田怡, 向清祥, 熊俊如, 张丽群, 李艳, 有机化学, 2013, 33, 2210.)

[30] Li, X.; Gao, X.; Shi, W.; Ma, H. Chem. Rev. 2014, 114, 590.

[31] Yang, Y.; Zhao, Q.; Feng, W.; Li, F. Chem. Rev. 2013, 113, 192.

[32] Lv, H. S.; Huang, S. Y.; Zhao, B. X.; Miao, J. Y. Anal. Chim. Acta 2013, 788, 177.

[33] Liu, X. D.; Xu, Y.; Sun, R.; Xu, Y. J.; Lu, J. M.; Ge, J. F. Analyst 2013, 138, 6542 .

[34] Lee, M. H.; Han, J. H.; Lee, J. H.; Park, N.; Kumar, R.; Kang, C.; Kim, J. S. Angew. Chem., Int. Ed. 2013, 52, 6206.

[35] McMahon, B. K.; Pal, R.; Parker, D. Chem. Commun. 2013, 49, 5363.

[36] Lv, H. S.; Liu, J.; Zhao, J.; Zhao, B. X.; Miao, J. Y. Sens. Actuators, B 2013, 177, 956 .

[37] Miao, F.; Song, G.; Sun, Y.; Liu, Y.; Guo, F.; Zhang, W.; Tian, M.; Yu, X. Biosens. Bioelectron. 2013, 50, 42.

[38] Chen, S.; Hong, Y.; Liu, Y.; Liu, J.; Leung, C. W. T.; Li, M.; Kwok, R. T. K.; Zhao, E.; Lam, J. W. Y.; Yu, Y.; Tang, B. Z. J. Am. Chem. Soc. 2013, 135, 4926.

[39] Xu, Y.; Jiang, Z.; Xiao, Y.; Bi, F. Z.; Miao, J. Y.; Zhao, B. X. Anal. Chim. Acta 2014, 820,146

[40] Sippel, T. O. J. Histochem. Cytochem. 1981, 29, 314.

[41] Yin, L. L.; Chen, Z. Z.; Tong, L. L.; Xu, K. H.; Tang, B. Chin. J. Anal. Chem. 2009, 37, 1073 (in Chinese).

(尹伶灵, 陈蓁蓁, 佟丽丽, 徐克花, 唐波, 分析化学, 2009, 37, 1073.)
[42] Jung, H. S.; Chen, X. Q.; Kim, J. S.; Yoon, J. Chem. Soc. Rev. 2013, 42, 6019.

[43] Yin, C. X.; Huo, F. J.; Zhang, J. J.; Martínez-Máñez, R.; Yang, Y. T.; Lv, H. G.; Li, S. D. Chem. Soc. Rev. 2013, 42, 6032.

[44] Chen, X. Q.; Zhou, Y.; Peng, X. J.; Yoon, J. Chem. Soc. Rev. 2010, 39, 2120.

[45] Kanaoka, Y.; Machida, M.; Ando, K.; Sekine, T. Biochim. Biophys. Acta 1970, 207, 269.

[46] Youziel, J.; Akhbar, A. R.; Aziz, Q.; Smith, M. E. B.; Caddick, S.; Tinker, A.; Baker, J. R. Org. Biomol. Chem. 2014, 12, 557.

[47] Liu, X. D.; Sun, R.; Xu, Y.; Xu, Y. J.; Ge, J. F.; Lu, J. M. Sens. Actuators, B 2013, 178, 525.

[48] Zhou, X.; Jin, X. J.; Sun, G. Y. Wu, X. Chem. Eur. J. 2013, 19, 7817.

[49] Yang, Y. T.; Huo, F. J.; Yin, C. X.; Zheng, A. M.; Chao, J. B.; Li, Y. Q.; Nie, Z. X.; Martínez-Máñez, R.; Liu, D. S. Biosens. Bioelectron. 2013, 47, 300.

[50] Wu, Q. Q.; Xiao, Z. F.; Du, X. J.; Song, Q. H. Chem. Asian J. 2013, $8,2564$.

[51] Wang, H. L.; Zhou, G. D.; Mao, C.; Chen, X. Q. Dyes Pigm. 2013, 96, 232.

[52] Long, L. L.; Zhou, L. P.; Wang, L.; Meng, S. C.; Gong, A. H.; Du, F. Y.; Zhang, C. Org. Biomol. Chem. 2013, 11, 8214.

[53] Zhang, M.; Yu, M. X.; Li, F. Y.; Zhu, M. W.; Li, M. Y.; Gao, Y. H.; Li, L.; Liu, Z. Q.; Zhang, J. Q.; Zhang, D. Q.; Yi, T.; Huang, C. H. J. Am. Chem. Soc. 2007, 129, 10322.

[54] Niu, L. Y.; Guan, Y. S.; Chen, Y. Z.; Wu, L. Z.; Tung, C. H.; Yang, Q. Z.J.Am. Chem. Soc. 2012, 134, 18928.

[55] Zhang, X. L.; Zhu, B. C.; Jia, H. Y.; Li, Y. M. Dyes Pigm. 2012, 94, 10.

[56] Tang, Y.; Yang, H. R.; Sun, H. B.; Liu, S. J.; Wang, J. X.; Zhao, Q.; Liu, X. M.; Xu, W. J.; Li, S. B.; Huang, W. Chem. Eur. J. 2013, 19, 1311.

[57] Maity, D.; Govindaraju, T. Org. Biomol. Chem. 2013, 11, 2098.

[58] Chen, S.; Hou, P.; Zhou, B.; Song, X.; Wu, J.; Zhang, H.; Foley, W. J. $R S C A d v$. 2013, 3, 11543 .

[59] Li, J.; Zhang, C. F.; Ming, Z. Z.; Yang, W. C.; Yang, G. F. RSC Adv. 2013, 3, 26059.

[60] Wei, M. J.; Yin, P.; Shen, Y. M.; Zhang, L. L.; Deng, J. H.; Xue, S. Y.; Li, H. T.; Guo, B.; Zhang, Y. Y.; Yao, S. Z. Chem. Commun. 2013, 49, 4640.

[61] Wang, S. Q.; Wu, Q. H.; Wang, H. Y.; Zheng, X. X.; Shen, S. L.; Zhang, Y. R.; Miao, J. Y.; Zhao, B. X. Analyst 2013, 138, 7169.

[62] Zhu, B. C.; Zhang, X. L.; Li, Y. M.; Wang, P. F.; Zhang, H. Y.; Zhuang, X. Q. Chem. Commun. 2010, 46, 5710.

[63] Lim, S. Y.; Na, M. J.; Kim, H. J. Sens. Actuators, B 2013, 185, 720.

[64] Fan, Z.; Wu, J.; Liu, W.; Ma, J.; Sun, J.; Wang, P. Tetrahedron 2013, 69, 4536.

[65] Yuan, L.; Lin, W. Y.; Chen, H.; Zhu, S. S.; He, L. W. Angew. Chem., Int. Ed. 2013, 52, 10018

[66] Wang, R.; Chen, L. X.; Liu, P.; Zhang, Qin.; Wang, Y. Q. Chem. Eur. J. 2012, 18, 11343.

[67] Zhai, D. T.; Lee, S. C.; Yun, S. W.; Chang, Y. T. Chem. Commun. 2013, 49, 7207.

[68] Das, Priyadip.; Mandal, A. K.; Reddy, G. U.; Baidya, M.; Ghosh, S. K.; Das, A. Org. Biomol. Chem. 2013, 11, 6604.

[69] Li, G. Y.; Chen, Y.; Wu, J. H.; Ji, L. N.; Chao, H. Chem. Commun. 2013, 49, 2040.

[70] Wang, H. L.; Zhou, G. D.; Chen, X. Q. Sens. Actuators, B 2013, $176,698$.

[71] Yang, X. F.; Guo. Y. X.; Strongin, R. M. Angew. Chem., Int. Ed. 2011, 50, 10690 
[72] Guo, Z. Q.; Nam, S. W.; Park, S.; Yoon, J. Chem. Sci. 2012, 3, 2760 .

[73] Xiong, X. Q.; Song, F. L.; Chen, G. W.; Sun, W.; Wang, J. Y.; Gao, P.; Zhang, Y. K.; Qiao, B.; Li, W. F.; Sun, S. G.; Fan, J. L.; Peng, X. J. Chem. Eur. J. 2013, 19, 6538.

[74] Wang, S. Q.; Wu, Q. H.; Wang, H. Y.; Zheng, X. X.; Shen, S. L.; Zhang, Y. R.; Miao, J. Y.; Zhao, B. X. Biosens. Bioelectron. 2014, $55,386$.

[75] Dai, X.; Wu, Q. H.; Wang, P. C.; Tian, J.; Xu, Y.; Wang, S. Q.; Miao, J. Y.; Zhao, B. X. Biosens. Bioelectron. 2014, 59, 35.
[76] Wang, P.; Liu, J.; Lv, X.; Liu, Y. L.; Zhao, Y.; Guo, W. Org. Lett. 2012, 14, 520.

[77] Kong, F. P.; Liu, R. P.; Chu, R. R.; Wang, X.; Xu, K. H.; Tang, B. Chem. Commun. 2013, 49, 9176.

[78] Yuan, L.; Lin, W. Y.; Yang, Y. T. Chem. Commun. 2011, 47, 6275.

[79] Ma, L. M.; Qian, J. H.; Tian, H. Y.; Lan, M. B.; Zhang, W. B. Analyst 2012, 137, 5046.

[80] Liu, J.; Sun, Y. Q.; Huo, Y.; Zhang, H.; Wang, L.; Zhang, P.; Song, D.; Shi, Y.; Guo, W. J. Am. Chem. Soc. 2014, 136, 574.

(Qin, X.) 